\title{
A NOTE ON SOME INEQUALITIES
}

\author{
by T. M. FLETT
}

(Received 17th May, 1958)

1. In the course of some recent work on Fourier series $[5,6]$ I had occasion to use a number of integral inequalities which were generalizations or limiting cases of known results. These inequalities may perhaps have other applications, and it seems worth while to collect them together in a separate note with one or two further results of a similar nature.

For any number $k$, used as an index (exponent), and such that $k>1$, we write $k^{\prime}=k /(k-1)$, so that $k$ and $k^{\prime}$ are conjugate indices in the sense of Hölder's inequality.

We use $B$ to denote a positive constant depending on the parameters $c, d, \ldots$ concerned in the particular problem in which it appears. If we wish to express the dependence explicitly, we write $B$ in the form $B(c, d, \ldots)$. We use $A$ to denote a positive absolute constant. These constants are not necessarily the same on any two occurrences. We also use suffixes to distinguish particular $B$ 's which retain their identity throughout.

Inequalities of the form

$$
L \leqslant B(c, d, \ldots) R
$$

are to be interpreted as meaning " if the expression $R$ is finite, then the expression $L$ is finite and satisfies the inequality".

2.1. The first of our inequalities is the following generalization of Hardy's inequality. $\dagger$

Theorem 1. Let $f(t) \geqslant 0$ in $t \geqslant 0$, and let

$$
F(t)=\int_{0}^{t} f(u) d u \quad(\gamma>-1), \quad F(t)=\int_{t}^{\infty} f(u) d u \quad(\gamma<-1) .
$$

If $q \geqslant p \geqslant 1$ and $\gamma \neq-1$, then

$$
\left\{\int_{0}^{\infty} t^{-1-\alpha \gamma}\left(\frac{F}{t}\right)^{q} d t\right\}^{1 / q} \leqslant B\left\{\int_{0}^{\infty} t^{-1-\nu \gamma} f^{p} d t\right\}^{1 / p}
$$

The case $q=p$ of this inequality is due to Hardy [8], while the case $q>p>1, \gamma=-1 / p$ is due to Hardy and Littlewood [9]. Hardy and Littlewood also conjectured the exact value of the constant $B$ in this latter case, and their conjecture was proved by Bliss [1]. The complete result above is stated without proof in [6].

The case $\gamma<-1$ of Theorem 1 is an easy deduction from the case $\gamma>-1$. For if $\gamma<-1$, and if

$$
t=\frac{1}{x} \text { and } \frac{1}{x^{2}} f\left(\frac{1}{x}\right)=g(x)
$$

then

$$
F^{\prime}(t)=\int_{t}^{\infty} f(u) d u=\int_{0}^{x} \frac{1}{v^{2}} f\left(\frac{1}{v}\right) d v=\int_{0}^{x} g(v) d v=G(x),
$$

say. Applying (2.1.1) to $g$ (with $\gamma$ replaced by $-\gamma-2>-1$ ), we obtain

† Hardy's inequality is the case $q=p>1, \gamma=-1 / p$ of Theorem 1 (see Hardy, Littlewood, and Pólya [14, Theorem 327]). 


$$
\begin{gathered}
\left\{\int_{0}^{\infty} t^{-1-q \gamma}\left(\frac{F(t)}{t}\right)^{q} d t\right\}^{1 / q}=\left\{\int_{0}^{\infty} x^{-1+q \gamma+2 q}\left(\frac{G(x)}{x}\right)^{q} d x\right\}^{1 / q} \leqslant B\left\{\int_{0}^{\infty} x^{-1+p(\gamma+2)} g^{p}(x) d x\right\}^{1 / p} \\
=B\left\{\int_{0}^{\infty} t^{-1-p \gamma} f^{p}(t) d t\right\}^{1 / p},
\end{gathered}
$$

and this is the result of the theorem for $f . \dagger$

To prove the inequality (2.1.1) when $\gamma>-1$, we may reduce the general case $q \geqslant p$ to the special case $q=p$ considered by Hardy. Alternatively, we may generalize the inequality by introducing fractional integrals, and then we obtain the following theorem.

THEOREm 2. Let $f(t) \geqslant 0$ in $t \geqslant 0$, and let $F_{\alpha}(t)$ be the $\alpha$-th Riemann-Liouville integral of $f$ with origin 0 , i.e.

$$
F_{\alpha}(t)=\frac{1}{\Gamma(\alpha)} \int_{0}^{t}(t-u)^{\alpha-1} f(u) d u .
$$

If either $q \geqslant p \geqslant 1$ and $\alpha>\frac{1}{p}-\frac{1}{q}$, or $q>p>1$ and $\alpha=\frac{1}{p}-\frac{1}{q}$, and if $\gamma>-1$, then

$$
\left\{\int_{0}^{\infty} t^{-1-q \gamma}\left(\frac{F_{\alpha}}{t^{\alpha}}\right)^{q} d t\right\}^{1 / q} \leqslant B\left\{\int_{0}^{\infty} t^{-1-p \gamma} f^{\nu} d t\right\}^{1 / p} .
$$

Various cases of this theorem are known. Thus the case $q>p>1, \gamma=-1 / p$ is due to Hardy and Littlewood [10, Theorem 4], the case $q=p>1, \gamma=-1 / p$ to Knopp $\$$ [15], and the case $q=p=1, \gamma \geqslant 0$ to Bosanquet [2]. Hardy and Littlewood have given a proof of a result [10, Theorem 7] which implies (2.1.2) for $-1<\gamma<0$, but their proof here appears to be incomplete. $\$$ They have also stated the case $q \geqslant p \geqslant 1, \gamma=0$ as the integral analogue of an inequality for series [13, Theorem 5].

The general result (2.1.2) can be proved by appropriate specialization of an argument used by Hardy and Littlewood [12] to prove an inequality for series. However, in one of our later applications we require an explicit value for the constant $B$ in one particular case of (2.1.2), and it seems advisable therefore to give the proof of Theorem 2 in full.|l

2.2. Suppose first that $q \geqslant p>1$. Let

$$
J=\left\{\int_{0}^{\infty} t^{-1-p \gamma} f^{p} d t\right\}^{1 / p}
$$

let $\alpha>1 / p-1 / q$, and let $\lambda$ and $\eta$ be numbers, to be chosen later, such that $\lambda<1 / p^{\prime}, 0<\eta<1$, and $p^{\prime}(\alpha-1)(1-\eta)>-1$. Applying Hölder's inequality with indices $q, p^{\prime}$, and $p q /(q-p)$, we have II

$$
F_{\alpha}(t)=\frac{1}{\Gamma(\alpha)} \int_{0}^{t}(t-u)^{\alpha-1} f(u) d u
$$

† This argument is given by Hardy [8].

† See also Hardy, Littlewood, and Pólya [14], Theorem 329.

$\$$ Their Theorem 7 depends on their Theorem 6 , and the discussion of the region $A^{\prime}$ in part (i) of the proof of the latter theorem does not seem to be valid.

\|I I have used this same argument in [7] to prove the series analogue of Theorem 2 . but have not attempted there to determine an explicit value for the constant.

II The last factor in $(2.2 .1)$ is to be omitted if $p=q$. 


$$
\begin{aligned}
& =\frac{1}{\Gamma(\alpha)} \int_{0}^{\ell}\left\{(t-u)^{(\alpha-1) \eta} u^{\lambda+(1+p \gamma)(q-p) /(p q)} f^{p / \alpha}(u)\right\}\left\{(t-u)^{(\alpha-1)(1-\eta)} u^{-\lambda}\right\}\left\{u^{-1-p \gamma} f^{p}(u)\right\}^{(q-p) /(p q)} d u \\
& \leqslant \frac{1}{\Gamma(\alpha)}\left\{\int_{0}^{t}(t-u)^{q(\alpha-1) \eta} u^{q \lambda+(1+p \gamma)(q-p) / p} f^{p}(u) d u\right\}^{1 / q}\left\{\int_{0}^{t}(t-u)^{p^{\prime}(\alpha-1)(1-\eta)} u^{-p^{\prime} \lambda} d u\right\}^{1 / p^{\prime}} \\
& \times\left\{\int_{0}^{t} u^{-1-p \gamma} f^{p}(u) d u\right\}^{(q-p) /(p q)} \\
& \leqslant B_{1} J^{1-p^{\prime} q} t^{(\alpha-1)(1-\eta)-\lambda+1 / p^{\prime}}\left\{\int_{0}^{t}(t-u)^{q(\alpha-1) \eta} u^{q \lambda+(1+p \gamma)(q-p) / p} f^{p}(u) d u\right\}^{1 / \eta},
\end{aligned}
$$

where

$$
B_{1}=\frac{1}{\Gamma(\alpha)}\left\{\int_{0}^{1}(1-v)^{p^{\prime}(\alpha-1)(1-\eta)} v^{-p^{\prime} \lambda} d v\right\}^{1 / p^{\prime}} .
$$

Write now $\mu=q(\alpha-1) \eta$ and $\omega=q(\lambda+\gamma+1 / p)$. Then we have

whence

$$
t^{-1-q \gamma-q \alpha} F_{\alpha}^{q}(t) \leqslant B_{1}^{q} J^{q-p} t^{-1-\mu-\omega} \int_{0}^{t}(t-u)^{\mu} u^{\omega-1-p \gamma} f^{p}(u) d u,
$$

where

$$
\int_{0}^{\infty} t^{-1-q v}\left(\frac{F_{\alpha}}{t^{\alpha}}\right)^{q} d t \leqslant B_{1}^{q} J^{q-p} \int_{0}^{\infty} u^{-1-p \gamma} f^{p}(u) k(u) d u
$$

$$
k(u)=u^{\omega} \int_{u}^{\infty} t^{-1-\mu-\omega}(t-u)^{\mu} d t=\int_{0}^{1} v^{-1+\omega}(1-v)^{\mu} d v=B_{2}, \text { say } .
$$

Thus, provided that $\omega>0$ and $\mu>-1$, we obtain (2.1.2) with $B=B_{1} B_{2}^{1 / q}$. Choose $\eta$ so that $q \eta=p^{\prime}(1-\eta)$, i.e. so that $\eta=p^{\prime} /\left(p^{\prime}+q\right)$. Then $0<\eta<1$ and

$$
\alpha-1>\frac{1}{p}-\frac{1}{q}-1=-\left(\frac{1}{p^{\prime}}+\frac{1}{q}\right)=-\frac{1}{q \eta}=-\frac{1}{p^{\prime}(1-\eta)},
$$

as required. Choose also $\lambda$ so that $\lambda<1 / p^{\prime}$ and $\omega=q(\lambda+\gamma+1 / p)>0$ (this is possible, since $\gamma+1>0)$. The various conditions which we have imposed on $\lambda$ and $\eta$ are therefore satisfied, and this completes the proof in this case. A similar argument applies if $q \geqslant p=1, \alpha>1 / p-1 / q$. (Take $\lambda=0, \eta=1$, and omit the second bracketed factor in (2.2.1). )

2.3. We digress for a moment to determine a value for the constant $B$ in (2.1.2) when $q>p>1, \alpha=1-1 / q, \gamma=-1 / p$, and $p^{\prime}>q$. We are actually interested only in the order of magnitude of $B$ when $p \rightarrow 1$, and it is convenient to choose values of $\lambda$ and $\eta$ different from those given above. From (2.2.2) and (2.2.3) the constant $B$ is here given by

$$
B=B_{1} B_{2}^{1 / q}=\frac{1}{\Gamma(1-1 / q)}\left\{\frac{\Gamma\left(1-p^{\prime}(1-\eta) / q\right) \Gamma\left(1-p^{\prime} \lambda\right)}{\Gamma\left(2-p^{\prime}(1-\eta) / q-p^{\prime} \lambda\right)}\right\}^{1 / p^{\prime}}\left\{\frac{\Gamma(q \lambda) \Gamma(1-\eta)}{\Gamma(q \lambda+1-\eta)}\right\}^{1 / q},
$$

and $\lambda$ and $\eta$ have to satisfy the conditions

$$
0<p^{\prime} \lambda<1,0<\eta<1 \text {, and } p^{\prime}(1-\eta) / q<1 \text {. }
$$

Choose $\lambda$ and $\eta$ so that $p^{\prime} \lambda=p^{\prime}(1-\eta) / q=\frac{1}{2}$. Then the conditions (2.3.1) are satisfied (since $\left.q<p^{\prime}\right)$, and, by the duplication formula for the $\Gamma$-function, $\dagger$

† See, for example, Copson [3], \$ 9.23. 


$$
\begin{aligned}
& B=\frac{\pi^{1 / p^{\prime}}}{\Gamma(1-1 / q)}\left\{\frac{\Gamma^{2}\left(\frac{q}{2 p^{\prime}}\right)}{\Gamma\left(\frac{q}{p^{\prime}}\right)}\right\}^{1 / q}=\frac{\pi^{1 / p^{\prime}}}{\Gamma(1-1 / q)}\left\{\frac{\pi^{1 / 2} \Gamma\left(\frac{q}{2 p^{\prime}}\right)}{2^{q / p^{\prime}-1} \Gamma\left(\frac{q}{2 p^{\prime}}+\frac{1}{2}\right)}\right\}^{1 / q} \\
& \leqslant B(q) \Gamma^{1 / q}\left(\frac{q}{2 p^{\prime}}\right) \leqslant B(q)\left(\frac{2 p^{\prime}}{q}\right)^{1 / q} \leqslant \frac{B(q)}{(p-1)^{1 / q}},
\end{aligned}
$$

where the constants $B(q)$ depend only on $q$.

2.4. We return now to the remaining case of Theorem 2 , namely $q>p>1, \alpha=1 / p-1 / q$. Write

Then

$$
\Gamma(\alpha) F_{\alpha}(t)=\int_{0}^{t}(t-u)^{\alpha-1} f(u) d u=\int_{0}^{\underline{t} t}+\int_{t t}^{t}=G(t)+H(t)
$$

$$
G(t) \leqslant B t^{\alpha-1} \int_{0}^{\frac{1}{t} t} f d u \leqslant B t^{\alpha-1} \int_{0}^{t} f d u=B t^{\alpha-1} F_{1}(t),
$$

whence, by the case already proved,

$$
\left\{\int_{0}^{\infty} t^{-1-q \gamma}\left(\frac{G}{t^{\alpha}}\right)^{q} d t\right\}^{1 / q} \leqslant B\left\{\int_{0}^{\infty} t^{-1-\alpha \gamma}\left(\frac{F_{1}}{t}\right)^{q} d t\right\}^{1 / q} \leqslant B\left\{\int_{0}^{\infty} t^{-1-p \gamma} f^{p} d t\right\}^{1 / p}
$$

It is therefore now enough to prove that

$$
\left\{\int_{0}^{\infty} t^{-1-q \gamma}\left(\frac{H}{t^{\alpha}}\right)^{q} d t\right\}^{1 / q} \leqslant B\left\{\int_{0}^{\infty} t^{-1-p \gamma} f^{p} d t\right\}^{1 / p}
$$

To prove this, it is sufficient, by the converse of Hölder's inequality, to prove that

$$
\int_{0}^{\infty} t^{-1 / q-\gamma-\alpha} h H d t \leqslant B\left\{\int_{0}^{\infty} t^{-1-p \gamma} f^{p} d t\right\}^{1 / p}\left\{\int_{0}^{\infty} h^{q^{\prime}} d t\right\}^{t / q^{\prime}}
$$

for every $h$ belonging to $L^{q^{\prime}}(0, \infty)$. The integral on the left of $(2.4 .2)$ is equal to

$$
\begin{aligned}
\int_{0}^{\infty} t^{-1 / q-\gamma-\alpha} h(t) d t \int_{\frac{1}{1} t}^{t}(t & -u)^{\alpha-1} f(u) d u \\
& =\int_{0}^{\infty} \int_{\frac{1}{2} t}^{t} u^{-1 / p-\gamma} f(u) h(t) u^{1 / p+\gamma} t^{-1 / q-\gamma-\alpha}(t-u)^{\alpha-1} d u d t \\
& \leqslant \int_{0}^{\infty} \int_{\frac{1}{2} t}^{t} u^{-1 / p-\gamma} f(u) h(t)(t-u)^{\alpha-1} d u d t
\end{aligned}
$$

(since in the region of integration $u^{1 / p+\gamma} \leqslant B t^{1 / p+\gamma}=B t^{1 / q+\gamma+\alpha}$ ). Since $\alpha-1=1 / p+1 / q^{\prime}-2$, it follows by a well-known theorem of Hardy and Littlewood [10, Theorem 3] $\dagger$ that the last integral in (2.4.3) does not exceed the right-hand side of (2.4.2) (for some $B$ ). This completes the proof of (2.4.1), and of Theorem 2.

2.5. We note in passing that the case $q=p>1$ of Theorem 2 is a particular case of the following general inequality (Hardy, Littlewood, and Pólya [14, Theorem 319]). that

Theorem A. Suppose that $p>1$, that $K(t, u)$ is non-negative and homogeneous of degree - 1 ,

† See also Hardy, Littlewood, and Pólya [14], Theorem 332.

† The equality of the two integrals in (2.5.1) is a consequence of the homogeneity of $k$. 


$$
\int_{0}^{\infty} K(1, u) u^{-1 / p} d u=\int_{0}^{\infty} K(t, 1) t^{-1 / p^{\prime}} d t=k,
$$

and that $f(t) \geqslant 0$ in $t \geqslant 0$. Then

$$
\int_{0}^{\infty} d t\left(\int_{0}^{\infty} K(t, u) f(u) d u\right)^{p} \leqslant k^{p} \int_{0}^{\infty} f^{p} d t
$$

Taking $K(t, u)=t^{-1 / p-\gamma-\alpha} u^{1 / p+\gamma}(t-u)^{\alpha-1}$ in $0<u \leqslant t$, and 0 elsewhere, and observing that

$$
\int_{0}^{\infty} K(1, u) u^{-1 ! p} d u=\int_{0}^{1} u^{\gamma}(1-u)^{\alpha-1} d u=\frac{\Gamma(\alpha) \Gamma(\gamma+1)}{\Gamma(\alpha+\gamma+1)}
$$

when $\alpha>0$ and $\gamma>-1$, we obtain (2.1.2) with

$$
B=\frac{\Gamma(\alpha) \Gamma(\gamma+1)}{\Gamma(\alpha+\gamma+1)}
$$

2.6. The result of Theorem $2 \nmid$ enables us to fill a gap in a theorem of the author on fractional integrals. Let $f$ be periodic with period $2 \pi$ and integrable in $(-\pi, \pi)$, let

and for $t>0$ let

$$
\phi(t)=\Phi_{0}(t)=f(\theta+t)+f(\theta-t) \text { and } \Lambda_{1}(t)=t \phi(t)-\int_{0}^{t} \phi d u,
$$

and

$$
\Phi_{\alpha}(t)=\frac{1}{\Gamma(\alpha)} \int_{0}^{t}(t-u)^{\alpha-1} \phi(u) d u \quad(\alpha>0)
$$

$$
\Lambda_{\alpha}(t)=\frac{1}{\Gamma(\alpha-1)} \int_{0}^{t}(t-u)^{\alpha-2} \Lambda_{1}(u) d u \quad(\alpha>1)
$$

(so that $\Phi_{\alpha}$ and $\Lambda_{\alpha}$ are the $\alpha$ th and $(\alpha-1)$ th integrals of $\phi$ and $\Lambda_{1}$, respectively). Then we have

THEOREM 3. Let $f$ be periodic with period $2 \pi$ and integrable in $(-\pi, \pi)$, and let the mean value of $f$ over this interval be 0 . Let also

$$
f_{k, \alpha}(\theta)=\left\{\int_{0}^{\pi}|\chi(t)|^{k} t^{-1-k \alpha} d t\right\}^{1 / k}
$$

where either $\chi=\Phi_{\alpha}$ and $\alpha \geqslant 0$, or $\chi=\Lambda_{\alpha}$ and $\alpha \geqslant 1$. Then for $1<k \leqslant 2$ and $p>1$

$$
\left\{\int_{-\pi}^{\pi}|f|^{p} d \theta\right\}^{1 / p} \leqslant B\left\{\int_{-\pi}^{\pi} \mathcal{F}_{k, \alpha}^{p} d \theta\right\}^{1 / p}+B \int_{-\pi}^{\pi}|f| d \theta
$$

This is proved in [6] for $\chi=\Phi_{\alpha}$ and $\alpha>1 / k$ and for $\chi=\Lambda_{\alpha}$ and $\alpha>1+1 / k$. Since $\mathfrak{f}_{k, \beta} \leqslant B(k, \alpha, \beta) \mathfrak{f}_{k, \alpha}$ for $\beta>\alpha$ (by Theorem. 2), the complete result follows.

3.1. We pass next to some results which are substitutes for Theorems 1 and 2 when one (or more) of the parameters takes a limiting value. We have first

TheоRem 4. Let $f(t) \geqslant 0$ in $0 \leqslant t \leqslant a$, where $a$ is finite, and let $F_{\alpha}(t)$ be the ath RiemannLiouville integral of $f$ with origin 0 . If $q>1$ and $\alpha=1-1 / q$, then

$\dagger$ We require only the case $q=p>1$ considered in $\S 2.5$. 


$$
\left\{\int_{0}^{a} F_{\alpha}^{q} d t\right\}^{1 / q} \leqslant B \int_{0}^{a} f(\log +f)^{1 / a} d t+B
$$

where the B's depend only on $q$ and a.

This result is the substitute for Theorem 2 when all three of the parameters $p, \alpha, \gamma$ have their limiting values (i.e. $p=1, \alpha=1-1 / q, \gamma=-1$ ). Zygmund [18] has proved the corresponding result for the Weyl fractional integral, and has remarked that his proof can be modified to apply to the Riemann-Liouville integral. We give here an alternative proof of Theorem 4 which is somewhat simpler than that of Zygmund. $\dagger$

Setting $f=0$ in $t>a$, we see from $\S 2.3$ that when $1<p<\operatorname{Min}\left(q, q^{\prime}\right)$ and $\alpha=1-1 / q$,

$$
\left\{\int_{0}^{a} t^{-q / p^{\prime}} F_{\alpha}^{q} d t\right\}^{1 / q} \leqslant B(q)(p-1)^{-1 / q}\left\{\int_{0}^{a} f^{p} d t\right\}^{1 / p}
$$

Since $t^{-1 / y^{\prime}}>\operatorname{Min}\left(a^{-1}, 1\right)$ in $0 \leqslant t \leqslant a$, we have therefore

$$
\left\{\int_{0}^{a} F_{\alpha}^{q} d t\right\}^{1 / q} \leqslant B(a, q)(p-1)^{-1 / q}\left\{\int_{0}^{a} f^{p} d t\right\}^{1 / p}
$$

for $1<p<\operatorname{Min}\left(q, q^{\prime}\right)$ and $\alpha=1-1 / q$, and the result of Theorem 4 now follows immediately from the following extrapolation theorem.

THEOREM 5. Let $T$ be a transformation which transforms a real function integrable in $(0, a)$, where $a$ is finite, into a function measurable in $(0, a)$, and has the properties

(ii) for any real $f_{1}$ and $f_{2}$,

$$
|T(f)|=|T(-f)|
$$

$$
\left|T\left(f_{1}+f_{2}\right)\right| \leqslant\left|T\left(f_{1}\right)\right|+\left|T\left(f_{2}\right)\right|,
$$

(iii) for any infinite sequence of non-negative functions $f_{n}$,

$$
\left|T\left(\sum_{1}^{\infty} f_{n}\right)\right| \leqslant \sum_{1}^{\infty}\left|T\left(f_{n}\right)\right|
$$

(iv) there exist constants $k>0, p_{0}>1, q \geqslant 1$, and $C>0$, such that for every real $f$ of $L^{\nu}(0, a)$, where $1<p<p_{0}$,

$$
\left\{\int_{0}^{a}|T(f)|^{a} d t\right\}^{1 / q} \leqslant C(p-1)^{-k}\left\{\int_{0}^{a}|f|^{p} d t\right\}^{1 / p}
$$

Then, for any integrable $f$,

$$
\left\{\int_{0}^{a}|T(f)|^{a} d t\right\}^{1 / 4} \leqslant B \int_{0}^{a}|f|(\log +|f|)^{k} d t+B
$$

where the constants $B$ depend only on $k, p_{0}$, and $C$.

The case $q=1$ of this result is due to Yano [17]. $\neq$ The proof of the general case is similar to that given by Yano, but for the sake of completeness we give the proof here.

We may suppose $f \geqslant 2$, for, if this is not so, write

$$
f_{1}=\left\{\begin{array}{cl}
f+2 & \text { if } f \geqslant 0, \\
2 & \text { if } f<0,
\end{array} \quad f_{2}=\left\{\begin{array}{cl}
2 & \text { if } f \geqslant 0, \\
-f+2 & \text { if } f<0 .
\end{array}\right.\right.
$$

$\dagger$ In our proof we use only the relatively simple case $\alpha=1-1 / q, \gamma=-1 / p$ of Theorem 2 discussed in $\S \S 2.2-3$. Zygmund, on the other hand, makes use of the case $q>p>1, \alpha=1 / p-1 / q, \gamma=-1 / p$ of Theorem 2, which depends on a difficult theorem of Hardy and Littlewood (see $\$$ 2.4).

$\ddagger$ It is also implicit in work of Titchmarsh [16]. 
Then $f_{1} \geqslant 2, f_{2} \geqslant 2, f=f_{1}-f_{2}$, and in virtue of (i) and (ii) it is enough to prove the theorem for $f_{1}$ and $f_{2}$.

Suppose then that $f \geqslant 2$. Let $E_{n}$ be the subset of $(0, a)$ in which $n-1 \leqslant f<n$, and let $f_{n}$ be equal to $f$ in $E_{n}$ and to 0 elsewhere. Then, by (iii) and Minkowski's inequality,

$$
\left\{\int_{0}^{a}|T(f)|^{a} d t\right\}^{1 / q} \leqslant\left\{\int_{0}^{a}\left(\sum_{3}^{\infty}\left|T\left(f_{n}\right)\right|\right)^{q} d t\right\}^{1 / q} \leqslant \sum_{3}^{\infty}\left\{\int_{0}^{a}\left|T\left(f_{n}\right)\right|^{a} d t\right\}^{1 / q},
$$

both sides possibly being $+\infty$. If now we take $p=1+\delta / \log n$ in (iv), where $\delta$ is positive and so small that $1+\delta / \log n<p_{0}$ for $n \geqslant 3$, we obtain

$$
\begin{aligned}
\left\{\int_{0}^{a}\left|T\left(f_{n}\right)\right|^{a} d t\right\}^{1 / q} & \leqslant \frac{C}{(p-1)^{k}}\left\{\int_{0}^{a}\left|f_{n}\right|^{p} d t\right\}^{1 / p} \leqslant \frac{C}{(p-1)^{k}} n\left|E_{n}\right|^{1 / p} \\
& =C \delta^{-k} n(\log n)^{k}\left|E_{n}\right|^{1-\delta /(8+\log n)} \\
& \leqslant B n(\log n)^{k}\left|E_{n}\right|+B n^{-2}(\log n)^{k}
\end{aligned}
$$

where both $B$ 's are of the form $B(C, k, \delta)$ [to prove that

$$
\left|E_{n}\right|^{1-\delta /(8+\log n)} \leqslant B\left|E_{n}\right|+B n^{-3}
$$

consider the cases $\left|E_{n}\right| \geqslant 1 / n^{3}$ and $\left|E_{n}\right| \leqslant 1 / n^{3}$ separately]. Hence

$$
\begin{aligned}
\left\{\int_{0}^{a}|T(f)|^{q} d t\right\}^{1 / q} & \leqslant B \sum_{3}^{\infty} n(\log n)^{k}\left|E_{n}\right|+B \\
& \leqslant B \sum_{3}^{\infty}(n-1)\{\log (n-1)\}^{k}\left|E_{n}\right|+B \\
& \leqslant B \int_{0}^{a} f(\log +f)^{k} d t+B
\end{aligned}
$$

where again both $B$ 's are of the form $B(C, k, \delta)$, and this proves the theorem.

3.2. If we combine Theorem 4 with the case $p=q>1, \gamma=-1 / p$ of Theorem 2 , and observe that $\left(F_{\alpha}\right)_{\beta}=F_{\alpha+\beta}$ for $\alpha>0$ and $\beta>0$, we obtain the case $q>1$ of the following result, which was stated without proof in [6].

Тнеовем 6. Let $f(t) \geqslant 0$ in $0 \leqslant t \leqslant a$, where $a$ is finite, and let $F_{1}(t)=\int_{0}^{t} f d u$. If $q \geqslant 1$, then

$$
\left\{\int_{0}^{a} t^{-1} F_{1}^{q} d t\right\}^{1 / q} \leqslant B_{3} \int_{0}^{a} f(\log +f)^{1 / a} d t+B_{4}
$$

where $B_{3}$ and $B_{4}$ are of the form $B(a, q)$.

This result is, of course, a substitute for Theorem 1 when $p=1$ and $\gamma=-1$. The case $q=1$ has been proved by Hardy and Littlewood [11] by an application of Young's inequality, and their method extends easily to give an alternative proof of the case $q>1$. Another proof of the case $q=1$ is given in [4], and this shows that in this case we may take the constants $B_{3}$ and $B_{4}$ to be of the form $A$ and $A a$, respectively.

3.3. We conclude this note by using the case $q=1$ of Theorem 6 to prove a substitute for Theorem $A$ in the case $p=1$. This was stated without proof in [5].

Throrem 7. Suppose that $K(t, u)$ is non-negative and homogeneous of degree -1, that 


$$
\sup _{0<u \leqslant 1} K(1, u)=k_{1}<\infty \text { and } \int_{0}^{1} K(t, 1) d t=k_{2}<\infty,
$$

and that $f(t) \geqslant 0$ in $(0, a)$, where $a$ is finite. Then

$$
\int_{0}^{a} d t\left\{\int_{0}^{a} K(t, u) f(u) d u\right\} \leqslant A\left(k_{1}+k_{2}\right)\left\{\int_{0}^{a} f \log +f d t+a\right\}
$$

and

$$
\left\{\int_{0}^{a} d t\left(\int_{0}^{a} K(t, u) f(u) d u\right)^{\mu}\right\}^{1 / \mu} \leqslant B\left(k_{1}, k_{2}, a, \mu\right) \int_{0}^{a} f d t \quad(0<\mu<1) .
$$

In addition to the result of Theorem 6 we require also the following inequality (Zygmund $[19, \S 10.22])$ : if $f \geqslant 0$ in $(0, a)$, where $a$ is finite, and if $0<\mu<1$, then

$$
\left\{\int_{0}^{a}\left(\frac{F_{1}}{t}\right)^{\mu} d t\right\}^{1 / \mu} \leqslant B(a, \mu) \int_{0}^{a} f d t .
$$

For $0<t \leqslant a$ we have

$$
\int_{0}^{a} K(t, u) f(u) d u=\frac{1}{t} \int_{0}^{t} t K(t, u) f(u) d u+\int_{t}^{a} K(t, u) f(u) d u=P(t)+Q(t),
$$

say. Writing $u=t v$, where $0<v \leqslant 1$, we have

$$
t K(t, u)=t K(t, t v)=K(1, v) \leqslant k_{1}
$$

so that

$$
P(t) \leqslant \frac{k_{1}}{t} \int_{0}^{t} f d u=k_{1}\left(\frac{F_{1}(t)}{t}\right) .
$$

Hence, by the case $q=1$ of Theorem 6 (with constants as determined in [4]) and (3.3.1),

and

$$
\int_{0}^{a} P d t \leqslant A k_{1} \int_{0}^{a} f \log +f d t+A a k_{1}
$$

Next, we have

$$
\left\{\int_{0}^{a} P^{\mu} d t\right\}^{1 / \mu} \leqslant B(a, \mu) k_{1} \int_{0}^{a} f d t
$$

for $0<\mu<1$, and

$$
\left\{\int_{0}^{a} Q^{\mu} d t\right\}^{1 / \mu} \leqslant B(a, \mu) \int_{0}^{a} Q d t
$$

$$
\begin{aligned}
\int_{0}^{a} Q d t & =\int_{0}^{a} f(u) d u \int_{0}^{u} K(t, u) d t=\int_{0}^{a} f(u) d u \int_{0}^{1} u K(u v, u) d v \\
& =\int_{0}^{a} f(u) d u \int_{0}^{1} K(v, 1) d v=k \int_{0} \int_{0}^{a} f(u) d u \\
& \leqslant A k_{2} \int_{0}^{a} f \log +f d u+k_{2} a,
\end{aligned}
$$

and the result now follows from these relations and the fact that

for $0<\mu \leqslant 1$.

$$
\int_{0}^{a} d t\left\{\int_{0}^{a} K(t, u) f(u) d u\right\}^{\mu} \leqslant \int_{0}^{a} p^{\mu} d t+\int_{0}^{a} Q^{\mu} d t
$$




\section{REFERENCES}

1. (i. A. Bliss, An integral inequality, J. London Math. Soc., 5 (1930), 40-46.

2. L. S. Bosanquet, The absolute summability (A) of Fourier series, Proc. Edinburgh Math. Soc. (2), 4 (1934), 12-17.

3. E. T. Copson, An introduction to the theory of functions of a complex variable (Oxford, 1935).

4. T. M. Flett, Some remarks on a maximal theorem of Hardy and Littlewood, Quart. J. of Math. (Oxford 2nd series), 6 (1955), 275-282.

5. T. M. Flett, Some theorems on odd and even functions, Proc. London Math. Soc. (3), 8 (1958), 135-148.

6. T. M. Flett. On the absolute summability of a Fourier series and its conjugate series, Proc. London Math. Soc. (3), 8 (1958), 258-311.

7. T. M. Flett, Some more theorems concerning the absolute summability of Fourier series and power series, Proc. London Math. Soc. (3), 8 (1958), 357-387.

8. G. H. Hardy, Notes on some points in the integral calculus, LXIV. Further inequalities between integrals, Messenger of Math., 57 (1927-8), 12-16.

9. G. H. Hardy and J. E. Littlewood, Notes on the theory of series (XII) : On certain inequalities connected with the calculus of variations, $J$. London Math. Soc., 5 (1930), 34-39.

10. G. H. Hardy and J. E. Littlewood, Some properties of fractional integrals. I, Math. Z., 27 (1928), 565-606.

11. G. H. . Hardy and J. E. Littlewood, A maximal theorem with function-theoretic applications, Acta Math., 54 (1930), 81-116.

12. G. H. Hardy and J. E. Littlewood, An inequality, Math. Z., 40 (1935), 1-40.

13. G. H. Hardy and J. E. Littlewood, Some more theorems concerning Fourier series and Fourier power series, Duke Math. J., 2 (1936), 354-382.

14. G. H. Hardy, J. E. Littlewood, and G. Pólya, Inequalities (Cambridge, 1934).

15. K. Knopp, Über Reihen mit positiven Gliedern (Zweite Mitteilung), J. London Math. Soc., $5(1930), 13-21$. 204-206.

16. E. C. Titchmarsh, Additional note on conjugate functions, J. London Math. Soc., 4 (1929),

17. S. Yano, Notes on Fourier analysis (XXIX): An extrapolation theorem, J. Math. Soc. Japan, 3 (1951), 206-305.

18. A. Zygmund, Some points in the theory of trigonometric and power series, Trans. Amer. Math. Soc., 36 (1934), 586-617.

19. A. Zygmund, Trigonometrical series (Warsaw, 1935).

Department of Pure Mathematics

The UNIVERSITY

LIVERPOOL 